Pacific Journal of Mathematics

ON REALIZING HIN GROUPS IN 3-MANIFOLDS 


\title{
ON REALIZING HNN GROUPS IN 3-MANIFOLDS
}

\author{
C. D. Feustel and R. J. Gregorac
}

\begin{abstract}
In this paper we suppose that the fundamental group of a 3-manifold $M$ has a presentation as an HNN group. We then show that under suitable conditions we can realize this presentation by embedding a closed, connected imcompressible surface in $M$.
\end{abstract}

In [2], [3], and [4] we show that if $\pi_{1}\left(M^{3}\right)$ is constructed in certain ways, one can realize this construction by a surface embedded in $M^{3}$. In this paper we show that one can realize the HNN construction when certain relationships between $\pi_{1}\left(M^{3}\right)$ and $M^{3}$ are present. The results in this paper are related to Theorem 2.4 in [10].

In this paper all spaces will be simplicial complexes, all maps will be piecewise linear, and all 3-manifolds will be 3-manifolds with boundary. However the boundary may be vacuous. Let $X$ be a connected subspace of a space $Y$. As usual we shall denote the boundary, closure, and interior of $X$ in $Y$ by bd $(X), \mathrm{cl}(X)$, and int $(X)$ respectively. The natural inclusion map from $X$ into $Y$ will be denoted by $\rho$ and the induced homomorphism from $\pi_{1}(X)$ into $\pi_{1}(Y)$ by $\rho_{*}$. Let $S$ be a closed connected surface other than the 2-sphere of projective plane embedded in a space $Y$. Then $S$ is incompressible in $Y$ if $\rho_{*}: \pi_{1}(S) \rightarrow \pi_{1}(Y)$ is one-to-one. If $S$ is a closed surface embedded in $Y$, then $S$ is incompressible in $Y$ if each component of $S$ is incompressible in $Y$. Irreducible and $P^{2}$-irreducible are defined as in [7]. We denote the unit interval $[0,1]$ by $I$ throughout.

Definition 1. Let $K$ be a group and $A$ a subgroup of $K$. Let $S$ be a closed connected surface other than the projective plane or 2-sphere. Let $A_{j} \cong \pi_{1}(S)$ and $A_{j} \subset A$ for $j=1,2$. Let $k$ be an element of $K$ not in $A$ such that $A_{1}=k^{-1} A_{2} k$. Then if $A$ and $k$ generate $K$ and all relations of $K$ are consequences of the relations of $A$ together with the relations $k$ induces between the elements of $A_{1}$ and $A_{2}$, we shall say that $K$ is an extension of $A$ by $k$ across $A_{1}$ and $A_{2}$. The reader will note that the class of groups defined above is a subclass of the Higmann, Neumann, Neumann (H.N.N.) groups [8].

Let $M$ be a 3-manifold, $x$ a point in $M$, and $S$ an incompressible surface in $M$ such that $M-S$ is connected. Then it is a consequence of Van Kampen's Theorem that $\pi_{1}(M, x)$ is an extension of $\pi_{1}(M-S, x)$ by some element of $\pi_{1}(M, x)$ across appropriate subgroups of $\pi_{1}(M, x)$. One might then wonder "If $\pi_{1}(M, x)$ were such an extension, could we embed in $M$ an incompressible surface which realizes this exten- 
sion." We will show blow that this can, in fact, be done. Let $M$ be a compact 3-manifold and $x$ a point of $M$. We suppose that $\pi_{1}(M, x)$ is an extension of $A$ by $k$ across $A_{1}$ and $A_{2}$ as given in Definition 1 above. We can represent this extension by an ordered sequence $\left\langle\pi_{1}(M, x), A, A_{1}, A_{2}, k\right\rangle$. If for each component $F$ of the boundary of $M$ some conjugate $\rho_{*} \pi_{1}(F)$ is contained in $A$, we shall say that the extension preserves the peripheral structure of $M$. Suppose a second representation of $\pi_{1}(M, x)$ is given by $\left\langle\pi_{1}(M, x), B, B_{1}, B_{2}, \hat{k}\right\rangle$ and this extension of $B$ is induced by an incompressible, closed, two-sided surface $S$ embedded in $M$ and a loop $l$ meeting $S$ in the single point $x$, i.e., $B$ is generated by the elements of $\pi_{1}(M, x)$ having representative loops which do not cross $S, \hat{k}=[l], B_{1}=\rho_{*} \pi_{1}(S, x)$ and $B_{2}=[l] B_{1}[l]^{-1}$. We shall say that $S$ realizes the extension of $B$ if there is an isomorphism

$$
\Phi: \pi_{1}(M, x) \longrightarrow \pi_{1}(M, x)
$$

such that

(1) $\Phi(A)=B$

(2) $\Phi\left(A_{j}\right)=B_{j} \quad j=1,2$

(3) $\Phi(k)=\hat{k}$.

THeOREM 1. Let $M$ be a compact 3-manifold such that $\pi_{2}(M)=0$. Let $S$ be a closed connected surface other than the 2-sphere or projective plane. Suppose $\pi_{1}(M, x)$ has a representation given by

$$
\left\langle\pi_{1}(M, x), A, A_{1}, A_{2}, k\right\rangle
$$

where $A_{1} \cong \pi_{1}(S)$ and the extension above preserves the peripheral structure of $M$. Then there is an embedding of $S$ in $M$ which realizes the given extension.

The proof of Theorem 1 above is similar in many respects to the proof of Theorem 1 in [3]. One first constructs a complex $X$ having the same fundamental group as $M$. One then finds a map $f: M \rightarrow X$ inducing an isomorphism from $\pi_{1}(M)$ to $\pi_{1}(X)$. The complex $X$ is constructed to contain an embedded surface $S$ realizing the given extension. One shows that there is a map $g$ homotopic to $f$ such that $g^{-1}(S)$ is an incompressible, connected, closed surface in $M$ and that $g^{-1}(S)$ realizes the given extension.

The following three lemmas appear in [4]. We omit the proofs which are not difficult.

Lemma 1. Let $M$ be a compact, connected 3-manifold such that $\pi_{2}(M)=0$. Let $X$ be a connected complex and $S$ a closed incompressi- 
ble surface embedded in $X$ and having a neighborhood homeomorphic to $S \times I$. We suppose that no component of $S$ is a 2-sphere or projective plane. Let $X_{k}, k=1, \cdots, n$ be the components of $X-S$. We suppose that $\pi_{i}(X)=\pi_{i}\left(X_{k}\right)=0$ for $i \geqq 2$ and $k=1, \cdots, n$. Let $f: M \rightarrow X$ be a map such that $f_{*}: \pi_{1}(M) \rightarrow \pi_{1}(X)$ is one-to-one $f \operatorname{bd}(M)$ does not meet $S$. Then there is a homotopy, constant on bd $(M)$, of $f$ to a map $g$ such that $g^{-1}(S)$ is an incompressible surface in $M$.

LEMMA 2. Let $S_{1}$ and $S_{2}$ be disjoint, incompressible, connected, two-sided surfaces which are embedded in a $P^{2}$-irreducible 3-maifold $M$. Then if $S_{1}$ is homotopic to $S_{2}$ in $M, S_{1} \cup S_{2}$ bounds an $S_{1} \times I$ embedded in $M$.

Lemma 3. Let $M_{1}$ be a compact, connected, 3-manifold, $X$ a connected complex, and $F$ and $S$ incompressible connected surfaces in $M_{1}$ and $X$ respectively. We suppose that $S$ is neither a 2-sphere or projective plane and $\pi_{i}(X)=0$ for $i \geqq 2$.

Let $f:\left(M_{1}, F\right) \rightarrow(X, S)$ be a map of pairs such that for some $x \in F$

$$
f_{*} \pi_{1}\left(M_{1}, x\right) \subset \pi_{1}(S, f(x)) \text {. }
$$

Then $f$ is homotopic under a deformation, constant on $F$, to a map into $S$.

Proof of Theorem 1. It is a consequence of Remark 1 in [9] that we may assume that $M$ is irreducible.

Let $\left(M_{A}, \hat{x}, p\right)$ be the covering space of $(M, x)$ associated with $A \subset$ $\pi_{1}(M, x)$. Let $f_{1}, f_{2} ;(S, y) \rightarrow(M, x)$ be maps such that $f_{j^{*}}\left(\pi_{1}(S, y)\right)=A_{j}$, for $j=1,2$. Since $f_{j^{*}}\left(\pi_{1}(S, y)\right) \subset p_{*} \pi_{1}\left(M_{A}, \hat{x}\right)$, there is a map $\hat{f}_{j}:(S, y) \rightarrow$ $\left(M_{A}, \hat{x}\right)$ such that $p \hat{f}_{j}=f_{j}$ for $j=1,2$. Let $X$ be the union of $M_{A}$ and $S \times I$ with identifications $\hat{f}_{1}(s)=(s, 0)$ and $\hat{f}_{2}(s)=(s, 1)$. We note that the arc $\{y\} \times[0,1] \subset S \times I$ becomes a simple loop $\hat{l}$ after the identification above since $\hat{f}_{1}(y)=\hat{f}_{2}(y)=\hat{x}$. Let $\Phi: A \cup\{k\} \rightarrow \pi_{1}(X, \hat{x})$ be a function defined by $\Phi(k)=[l]$ and $\Phi(a)=P_{*}^{-1}(a)$ for $a \in A$. Then $\Phi$ can be extended to an isomorphism of $\pi_{1}(M, x)$ onto $\pi_{1}(X, \hat{x})$ since $X$ has been constructed so that $\pi_{1}(X, \hat{x})$ will have a presentation identical to the given presentation of $\pi_{1}(M, x)$.

It can be shown as in the proof of the theorem in [2] that $\pi_{i}(X)=$ $\pi_{i}(X-S)=0$ for $i \geqq 2$.

We denotes $S \times\{1 / 2\} \subset X$ by $S$.

Let the boundary of $M$ be expressed as $\bigcup_{m=1}^{n} F_{m}$ where $F_{m}$ is a closed connected 2-manifold. Then some conjugate of $\rho_{*} \pi_{1}\left(F_{m}\right)$ is contained in $A$ for $m=1, \cdots, n$. Thus we can find a collection $\left\{\alpha_{m} \mid m=1, \cdots, n\right\}$ of simple arcs embedded in $M$ such that intersec- 
tion of each pair of these arcs is $x, \alpha_{m}$ meets $F_{m}$ in a single point, and there is a map $\hat{\rho}: \bigcup_{m=1}^{n}\left(F_{m} \cup \alpha_{m}\right) \rightarrow M_{A}$ such that $p \hat{\rho}=\rho$. Note that for each loop $l_{0}$ in $\bigcup_{m=1}^{n}\left(F_{m} \cup \alpha_{m}\right)$ based at $x,\left[\hat{\rho} l_{0}\right]=\Phi\left[l_{0}\right]$. Since $\hat{\rho}_{*} \rho_{*}=\Phi \rho_{*}: \pi_{1}\left(\bigcup_{m=1}^{n}\left(F_{m} \cup \alpha_{m}\right), x\right) \rightarrow \pi_{1}(X, \hat{x})$, we can extend $\hat{\rho}$ to a map $f: M \rightarrow X$ such that $\Phi=f_{*}: \pi_{1}(M, x) \rightarrow \pi_{1}(X, \hat{x})$ by using standard techniques from obstruction theory. (See [2] or [3] for the details of this construction.) It is a consequence of Lemma 1 that there is a map $g_{1}$ homotopic to $f$ such that $g_{1}^{-1}(S)$ is an incompressible surface in $M$ and $g_{1}=f$ on the boundary of $M$.

Since $g_{1}^{-1}(S)$ and $S$ are incompressible in $M$ and $X$ respectively, if $S_{0}$ is any component of $g_{1}^{-1}(S)$, the homomorphism $\left(g_{1} \mid S_{0}\right)_{*}: \pi_{1}\left(S_{0}\right) \rightarrow \pi_{1}(S)$ is one-to-one. Thus by Theorem 1 in [6] $g_{1} \mid S_{0}$ is homotopic to a covering map. Thus after a deformation, constant outside of a small neighborhood of $S_{0}$, we may assume that $g_{1} \mid S_{0}$ is a local homeomorphism. Thus we may assume that $g_{1}$ is a local homeomorphism on $g_{1}^{-1}(S)$.

Let $z$ be a point on $S_{0}$. Suppose that the isomorphism $\Phi_{0}=$ $g_{1 *}: \pi_{1}(M, z) \rightarrow \pi_{1}\left(X, g_{1}(z)\right)$ does not carry $\pi_{1}\left(S_{0}, z\right)$ onto $\pi_{1}\left(S, g_{1}(z)\right)$. It is a consequence of the result in [1] that $M$ is $P^{2}$-irreducible. Since $\Phi_{0}^{-1} \pi_{1}\left(S, g_{1}(z)\right)$ would properly contain $\pi_{1}\left(S_{0}, x\right)$, we would have by Theorem 6 in [7] that $S_{0}$ bounds a twisted line bundle $N \subset M$. One can easily show using the techniques of [7], as has been done in [5], that $\rho_{*} \pi_{1}(N, z)$ may be taken to be $\Phi_{0}^{-1}\left(\rho_{*} \pi_{1}\left(S, g_{1}(z)\right)\right)$. It follows from Lemma 3 that there is a deformation of $g_{1}$ to a map $g_{2}$ which pushes $g_{1}(N)$ first onto $S$ and then to one side of $S$ so that $g_{2}^{-1}(S)=g_{1}^{-1}(S)-S_{0}$. Thus we can assume that $\left(g_{1} \mid S_{0}\right)_{*}: \pi_{1}\left(S_{0}\right) \rightarrow \pi_{1}(S)$ is an epimorphism for each component $S_{0}$ of $g_{1}^{-1}(S)$.

Since $\pi_{1}(M) \not \subset A, g_{1}^{-1}(S)$ is not empty.

Let $S_{0}$ and $S_{1}$ be components of $g_{1}^{-1}(S)$. We claim that $S_{0} \cup S_{1}$ bounds a copy of $S_{0} \times[0,1]$ embedded in $M$. Since $M$ is $P^{2}$-irreducible, this will follow from Lemma 2 after we show that $S_{0}$ and $S_{1}$ are homotopic. Let $z_{0}$ be a point on $S_{0}$. Since $g_{1} \mid S_{0}$ and $g_{1} \mid S_{1}$ are assumed to be homeomorphisms, there is a unique point $z_{1}$ on $S_{1}$ such that $g_{1}\left(z_{0}\right)=g_{1}\left(z_{1}\right)$. Let $\alpha$ be an arc running from $z_{0}$ to $z_{1}$. Since $g_{1^{*}}$ is an isomorphism, we can find a loop $l_{1}$ based at $z_{0}$ such that the loops $g_{1}\left(l_{1}\right)$ and $g_{1}(\alpha)$ represent the same element in $\pi_{1}\left(X, g_{1}\left(z_{0}\right)\right)$. Thus we may assume that $\left[g_{1}(\alpha)\right]=1 \in \pi_{1}(X)$. Let $\lambda_{0}$ be a loop on $S_{0}$ based at $z_{0}$ and $\lambda_{1}$ a loop on $S_{1}$ such that $g_{1}\left(\lambda_{0}\right)=g_{1}\left(\lambda_{1}\right)$. Since the loop $g_{1}\left(\lambda_{0}\right) g_{1}(\alpha)\left(g_{1}\left(\lambda_{1}\right)\right)^{-1}\left(g_{1}(\alpha)\right)^{-1}$ is nullhomotopic and $\pi_{2}(X)=0$, we can show as in the proof of Theorem 1 in [3] that $S_{0}$ and $S_{1}$ are homotopic. Our claim follows.

We wish to show that we may assume $g_{1}^{-1}(S)$ contains exactly one component.

Suppose there is more than one component in $g_{1}^{-1}(S)$ and that the 
number of components of $g_{1}^{-1}(S)$ cannot be decreased by a small deformation of $g_{1}$. Let $l: S^{1} \rightarrow M$ be a loop in $M$ such that $g_{1^{*}}[l]=[\hat{l}]$. We may assume that

(i) $g_{1}(l)$ meets $S$ since the intersection number of [ $\left.\hat{l}\right]$ and $S$ is one. Thus we can take our basepoint to lie on one of the surfaces in $g_{1}^{-1}(S)$.

(ii) $l$ crosses $g_{1}^{-1}(S)$ at each point in $l \cap g^{-1}(S)$ and thus $\left(g_{1} l\right)^{-1}(S)$ is a finite set whose cardinality cannnot be reduced.

(iii) $g_{1}\left(l \cap g_{1}^{-1}(S)\right)$ is a single point.

Let $D$ be a disk and $\beta_{1}$ and $\beta_{2}$ arcs in the boundary of $D$ such that $\beta_{1} \cap \beta_{2}=$ bd $\left(\beta_{1}\right)$. Then we can define a map $\gamma: D \rightarrow X$ such that $\gamma\left(\beta_{1}\right)$ is the loop $g_{1} l\left(S^{1}\right)$ and $\gamma\left(\beta_{2}\right)$ is the loop $\hat{l}$.

We wish to show that $g_{1}^{-1}(S)$ may be taken to be homeomorphic to $S$ (connected). Assume that $g_{1}^{-1}(S)$ is not connected; then it has been shown that each pair of distinct surfaces in $g_{1}^{-1}(S)$ bounds a copy of $S \times I$ embedded in $M$. If this is the case, it is clear that $l^{-1} g_{1}^{-1}(S)$ contains more than one point. Let $\delta_{1}, \cdots, \delta_{v}$ be the closures of the components of $S^{1}-l^{-1} g_{1}^{-1}(S)$. After a general position argument we may assume $\gamma^{-1}(S)$ contains an arc $\beta_{3}$ which cuts off an arc $\beta_{4} \subset \beta_{1}$ and that $g_{1} l\left(\delta_{1}\right)=\gamma\left(\beta_{4}\right)$. Now $l$ carries bd $\left(\delta_{1}\right)$ to one or two components of $g_{1}^{-1}(S)$.

If $l\left(\mathrm{bd}\left(\delta_{1}\right)\right)$ is a single point, the loop $l\left(\delta_{1}\right)$ is homotopic to a loop $l_{1} \subset g_{1}^{-1}(S)$ such that $g_{1}\left(l_{1}\right)=\gamma\left(\beta_{3}\right)$ since the restriction of $g_{1}$ to each component of $g_{1}^{-1}(S)$ is a homeomorphism and $g_{1^{*}}$ is an isomorphism. It would follow that the number of points in $l^{-1} g_{1}^{-1}(S)$ could have been reduced by a different choice of $l$. Thus we conclude that $l$ carries the points of bd $\left(\delta_{1}\right)$ to distinct components of $g_{1}^{-1}(S)$.

Let $N$ be closure of the component of $M-g_{1}^{-1}(S)$ which meets $l\left(\delta_{1}\right)$. Let $S_{0}$ be a component of bd $(N)$. Since $g_{1} \mid S_{0}$ is a homeomorphism and the loop $g_{1} l\left(\delta_{1}\right)$ is homotopic to a loop in $S$, we may assume that the loop $g_{1} l\left(\delta_{1}\right)$ is homotopic to a point. (One alters the image of $l$ in a neighborhood of $S_{0}$ )

Since the loop $g_{1} l\left(\delta_{1}\right)$ is nullhomotopic in $X$, it can be shown that the map $g_{1} \mid N$ is homotopic $\bmod$ bd $(N)$ to a map into $S$; full details of a similar argument appear in [3]. It follows after an argument by induction that there exists a map $g: M \rightarrow X$ homotopic to $g_{1} \bmod$ bd $(M)$ such that $g^{-1}(S)$ contains exactly one component $S_{0}$ and $g \mid S_{0}$ is a homeomorphism. After an argument similar to the one given above, we can find a loop $l$ meeting $S_{0}$ in a single point and based at $x \in M$ such that $g_{*}[l]=[\hat{l}]$.

We observe that $S_{0}$ and $l$ induce an expression of $\pi_{1}(M, x)$ as an extension of a subgroup $B$ of $\pi_{1}(M, x)$. Let $B_{1}$ and $B_{2}$ be the associated subgroups of $\pi_{1}(M, x)$. Then we see that our map $g$ induces 
an isomorphism $g_{*}: \pi_{1}(M, x) \rightarrow \pi_{1}(M, x)$ such that

(1) $g_{*}(B) \subset A$

(2) $g_{*}\left(B_{1}\right)=A_{1}$

(3) $g_{*} B_{2}=A_{2}$.

Thus Theorem 1 is an immediate consequence of the remark preceding Lemma 2 on page 238 in [8] which shows that $g_{*}$ sends $B$ onto $A$.

REMARK 1. The remark mentioned above allows us to strengthen the statement of the theorem in [2] so that the splitting and the cutting are both actually realized.

REMARK 2. We can also realize geometrically more general presentations of $\pi_{1}(M)$ as an HNN group. In particular one might have that $\pi_{1}(M)$ has a presentation as in the first definition in $\S 4$ in [8] where each of the subgroups $L_{i}$ of $K$ is isomorphic to the fundamental group of a closed connected surface other than $S^{2}$ or the projective plane and there are only finitely many of the $t_{i}$. The proof of this result varies only slightly from the one given above.

REMARK 3. Theorem 1 in this paper together with Theorem 1 in [3] or [4] give us a sort of converse to Van Kampen's theorem as applied to a closed, connected, incompressible surface, other than $S^{2}$ or the projective plane, embedded in the interior of a compact 3manifold.

REMARK 4. This paper is in some sense a generalization of Stalling's work in [11].

\section{REFERENCES}

1. C. D. Feustel, $M^{3}$ admitting a certain embedding of $P^{2}$ is a pseudo $P^{3}$, Proc. Amer. Math. Soc., 26 (1970), 215-216.

2. - On replacing proper Dehn maps with proper embeddings, Trans. Amer. Math. Soc., 166 (1972), 261-267.

3. - A splitting theorem for closed orientable 3-manifolds, Topology, 11 (1972), 151-158.

4. - A generalization of Kneser's conjecture, Pacific J. Math., to appear.

5. - On S-maximal subgroups of $\pi_{1}\left(M^{2}\right)$, Canad. J. Math., 24 (1972), 439-449.

6. W. Heil, On P3-irreducible 3-manifolds, Bull. Amer. Math. Soc., 75 (1969), 772-775.

7. W. Jaco, Finitely presented subgroups of three-manifold groups, Inventions Math., 13 (1971), 335-346.

8. A. Karrass and D. Solitar, The subgroups of a free product of two groups with an amalgamated subgroups, Trans. Amer. Math. Soc., 150 (1970), 227-255.

9. J. Milnor, A unique decomposition theorem for 3-manifolds, Amer. J. Math., 84 (1962), 1-7. 
10. G. P. Scott, On sufficiently large 3-manifolds, Quart. J. Math. Oxford, 23 (1972), 159-172.

11. J. Stallings, On Fibering Certain 3-Manifolds, Topology of 3-manifolds, PrenticeHall, Englewood Cliffs, N. J., 1962, 95-100.

Received April 28, 1972 and in revised form October 3, 1972.

Virginia Polytechnic Institute and State University

AND

IOWA State UnIVERSITY 



\section{PACIFIC JOURNAL OF MATHEMATICS}

\section{EDITORS}

D. Gilbarg and J. Milgram

Stanford University

Stanford, California 94305

\author{
R. A. Beaumont \\ University of Washington \\ Seattle, Washington 98105
}

J. DuGUNDJI

Department of Mathematics

University of Southern California

Los Angeles, California 90007

Richard ARENS

University of California

Los Angeles, California 90024

\section{ASSOCIATE EDITORS}
E. F. BECKENBACH
B. H. NeumanN
F. WOLF
K. YosHIDA

\section{SUPPORTING INSTITUTIONS}

UNIVERSITY OF BRITISH COLUMBIA

CALIFORNIA INSTITUTE OF TECHNOLOGY

UNIVERSITY OF CALIFORNIA

MONTANA STATE UNIVERSITY

UNIVERSITY OF NEVADA

NEW MEXICO STATE UNIVERSITY

OREGON STATE UNIVERSITY

UNIVERSITY OF OREGON

OSAKA UNIVERSITY

\author{
UNIVERSITY OF SOUTHERN CALIFORNIA \\ STANFORD UNIVERSITY \\ UNIVERSITY OF TOKYO \\ UNIVERSITY OF UTAH \\ WASHINGTON STATE UNIVERSITY \\ UNIVERSITY OF WASHINGTON
* * * *
AMERICAN MATHEMATICAL SOCIETY \\ NAVAL WEAPONS CENTER
}

The Supporting Institutions listed above contribute to the cost of publication of this Journal, but they are not owners or publishers and have no responsibility for its content or policies.

Mathematical papers intended for publication in the Pacific Journal of Mathematics should be in typed form or offset-reproduced, (not dittoed), double spaced with large margins. Underline Greek letters in red, German in green, and script in blue. The first paragraph or two must be capable of being used separately as a synopsis of the entire paper. The editorial "we" must not be used in the synopsis, and items of the bibliography should not be cited there unless absolutely necessary, in which case they must be identified by author and Journal, rather than by item number. Manuscripts, in duplicate if possible, may be sent to any one of the four editors. Please classify according to the scheme of Math. Rev. Index to Vol. 39. All other communications to the editors should be addressed to the managing editor, Richard Arens, University of California, Los Angeles, California, 90024.

50 reprints are provided free for each article; additional copies may be obtained at cost in multiples of 50 .

The Pacific Journal of Mathematics is issued monthly as of January 1966. Regular subscription rate: $\$ 48.00$ a year (6 Vols., 12 issues). Special rate: $\$ 24.00$ a year to individual members of supporting institutions.

Subscriptions, orders for back numbers, and changes of address should be sent to Pacific Journal of Mathematics, 103 Highland Boulevard, Berkeley, California, 94708.

PUBLISHED BY PACIFIC JOURNAL OF MATHEMATICS, A NON-PROFIT CORPORATION

Printed at Kokusai Bunken Insatsusha (International Academic Printing Co., Ltd.), 270, 3-chome Totsuka-cho, Shinjuku-ku, Tokyo 160, Japan. 


\section{Pacific Journal of Mathematics}

\section{Vol. 46, No. 2 December, 1973}

Christopher Allday, Rational Whitehead products and a spectral sequence of

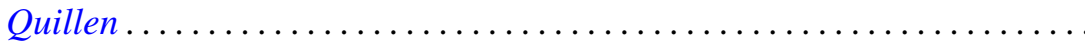

James Edward Arnold, Jr., Attaching Hurewicz fibrations with fiber

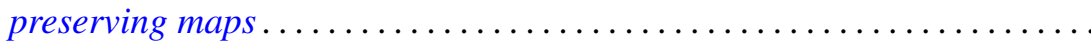

Catherine Bandle and Moshe Marcus, Radial averaging transformations with various metrics.................................

David Wilmot Barnette, A proof of the lower bound conjecture for convex

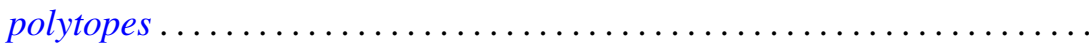

Louis Harvey Blake, Simple extensions of measures and the preservation of

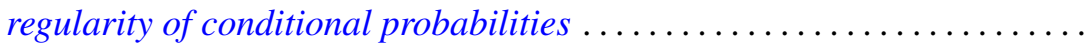

James W. Cannon, New proofs of Bing's approximation theorems for

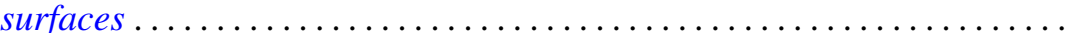

C. D. Feustel and Robert John Gregorac, On realizing HNN groups in

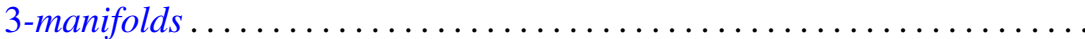

Theodore William Gamelin, Iversen's theorem and fiber algebras . . . . . . . . 389

Daniel H. Gottlieb, The total space of universal fibrations . . . . . . . . . . . .

Yoshimitsu Hasegawa, Integrability theorems for power series expansions of

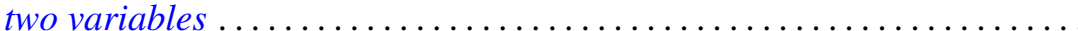

Dean Robert Hickerson, Length of period simple continued fraction expansion of $\sqrt{ } d$

Herbert Meyer Kamowitz, The spectra of endomorphisms of the disc algebra.

Dong S. Kim, Boundedly holomorphic convex domains

Daniel Ralph Lewis, Integral operators on $\mathscr{L}_{p}$-spaces ...

John Eldon Mack, Fields of topological spaces . . . . . . . . .

V. B. Moscatelli, On a problem of completion in bornology

Ellen Elizabeth Reed, Proximity convergence structures. .

Ronald C. Rosier, Dual spaces of certain vector sequence spaces .

Robert A. Rubin, Absolutely torsion-free rings

Leo Sario and Cecilia Wang, Radial quasiharmonic functions . .

James Henry Schmerl, Peano models with many generic classes .

H. J. Schmidt, The $\mathscr{F}$-depth of an $\mathscr{F}$-projector ............

Edward Silverman, Strong quasi-convexity. . . . . . . . . . . . . . . . . 549

Barry Simon, Uniform crossnorms ....................... 555

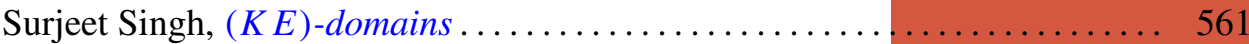

Ted Joe Suffridge, Starlike and convex maps in Banach spaces . . . . . . . . 575

Milton Don Ulmer, $C$-embedded $\Sigma$-spaces . . . . . . . . . . . . . . . . 591

Wolmer Vasconcelos, Conductor, projectivity and injectivity . . . . . . . . . 603 